\title{
Immune dysfunction in the presence of residual splenic tissue
}

\author{
R C COHEN AND A FERRANTE
}

Department of Paediatric Surgery and Department of Paediatrics, University of Adelaide, Adelaide Children's Hospital, Australia

SUMMARY Immunological function was examined in children who had undergone splenectomy, in 8 for trauma, and in 11 for haematologic/oncologic reasons. Particular emphasis was placed on the effects of residual splenic tissue on immune function. Children in the elective group had no evidence of splenosis but 6 of the 8 trauma patients showed residual splenic activity. A general trend indicated that immunological dysfunction was associated with the presence of residual splenic tissue. Three patients with significant post traumatic splenosis showed low IgM levels, one also had a low IgG level and another a low IgA and impaired lymphocyte response to mitogens. The trauma patients with little or no splenic tissue had normal immune functions. Immunological abnormalities were found in 8 of the 11 haematologic/oncologic patients with no splenosis suggesting the abnormalities were possibly due to the primary disease. In contrast to the popular belief that splenosis confers protection against overwhelming sepsis, the present findings suggest that patients with residual splenic tissue are at a greater risk of infection because of a lower level of immune response.

The spleen plays an important role in host resistance to infection. ${ }^{1}$ Among the many properties of splenic tissue are the elaboration of specific immune responses and clearance of micro-organisms, and old or injured red blood cells from the circulation. ${ }^{2} 3$ Removal of the spleen either for trauma or for haematologic indications is well known to be associated with an increased incidence of morbidity due to sepsis. ${ }^{45}$ The risk of this is lowest after splenectomy for trauma ${ }^{4}$ and it has been suggested that the common occurrence of post-traumatic splenosis may provide protection against overwhelming sepsis in such children. ${ }^{6}$ However, clinical evidence of morbidity and mortality from sepsis in patients with well documented evidence of splenosis would seem to cast doubt on the protective nature of residual splenic tissue. ${ }^{4-9}$ In the present study we compared the immune function of patients with and without residual splenic tissue after splenectomy for either traumatic or haematologic indications.

\section{Patients and methods}

The study comprised 18 children who underwent splenectomy at Adelaide Children's Hospital for either trauma or haematologic indications and one boy, aged 9 years, who had a complete splenic avulsion injury diagnosed on nuclear scan and treated non-operatively in 1980 . Seven patients (4 boys and 3 girls) aged 8 to 13 years underwent splenectomy during the period 1966-75 for blunt splenic trauma. At laparotomy all had either lacerated or transected spleens. At the time of restudy their ages ranged from 10 to 24 years. The remaining 11 patients ( 6 boys and 5 girls) aged 3 to 13 years were also studied. These patients underwent elective splenectomy during the period $1970-81$ for haematologic indications ( 3 for thalassaemia major, 4 for hereditary spherocytosis, 2 for idiopathic thrombocytopenic purpura, and 2 for staging of Hodgkin's disease). The ages of these patients at restudy were 6 to 32 years. The 2 patients with Hodgkin's disease were chosen because they had completed their course of treatment at least 3 years previously. One of these was stage $1 \mathrm{~A}$ treated with local radiotherapy, the other was stage IVA treated with radiotherapy and chemotherapy. Each thalassaemic patient had a routine whole blood transfusion one month before being investigated.

\section{Methods for determining residual splenic tissue}

Two methods were used to detect residual splenic tissue: ${ }^{99 \mathrm{~m} T c}$ phytate liver/spleen scan and assessing the percentage of circulating 'pitted' red cells by 
interference phase microscopical examination. Venous blood was taken from all patients just before performing a nuclear liver/spleen scan, so that only one venepuncture was required. Blood was taken from 10 normal control children with no known haematologic, splenic, or hepatic disease. The blood was treated according to the method of Holroyde et $a l .{ }^{10}$ One drop of blood was immediately placed into $0.5 \mathrm{ml}$ of phosphate buffered glutaraldehyde solution, $\mathrm{pH}$ 7.4. This was examined as a wet preparation with oil objective lens on an Olympus interference phase contrast microscope using Nomarski optics $(\times 1250) ; 2000$ cells were individually scanned on two separate specimens from each patient to determine the percentage of 'pitted' red cells.

The liver/spleen scans were carried out using a Searle Pho Gamma IV Gamma camera, fitted with either a low-energy all-purpose, or diverging collimator as appropriate to the patient's size. Each patient was injected intravenously with $70 \mu \mathrm{Ci} / \mathrm{kg}$ ${ }^{99} \mathrm{~m}$ technetium phytate. Images were obtained about 10 minutes after injection when the maximum liver/spleen uptake of the radionuclide occurred.

Routine liver/spleen views were performed including anterior, right lateral, posterior, left posterior oblique, and left anterior oblique positions. Anterior views of the pelvis and abdomen were also obtained to identify the presence and site of possible splenoses. Each scan took about 30 minutes.

In one case, where difficulty was encountered in visually separating residual splenic tissue from the left lobe of the liver, scanning was done using autologous, technetium labelled, heat affected red cells.

We found it difficult to assign accurate volumes to the residual splenic tissue. Using simple geometrical calculations we looked at a range of normal spleens and found that the calculations yielded volumes which correlated well with spleen weights from necropsy studies. ${ }^{11}$ In most of our cases, the residual spleen was clearly spherical which made calculation easy and probably reasonably accurate. With other shapes of residual spleen we used the geometrical models which seemed appropriate. We related our calculated volumes to the volume we regarded as normal for the patient's age, and expressed the volume of residual splenic tissue as a percentage of normal.

\section{Immune function studies}

Mononuclear leucocytes (MNL) and polymorphonuclear leucocytes (PMNL) were prepared using the method of Ferrante and Thong. ${ }^{12}$ Briefly, heparinised blood samples were layered on to Ficoll-Hypaque medium density $1.114 \mathrm{~g} / \mathrm{ml}$. After centrifugation for $30 \mathrm{~min}$, the MNL and PMNL were recovered as distinct cellular fractions at the interface and between the interface and the sedimented erythrocytes respectively. The cell populations both of $>95 \%$ purity were washed three times in medium 199 .

The percentage of T- and B-cells in the MNL fraction was enumerated by the E-rosette technique ${ }^{13}$ and binding of FITC-labelled goat anti-immunoglobulin. ${ }^{14}$ Lymphocyte transformation studies were performed as previously described. ${ }^{12}$ Briefly, to each well of a microtitre plate was seeded $2 \times 10^{5}$ lymphocytes (MNL) in $0 \cdot 1 \mathrm{ml}$ RPMI 1640 medium, supplemented with $10 \%$ heat-inactivated fetal calf serum. Lymphocytes were stimulated by addition of either $0.1 \mathrm{ml}$ phytohaemagglutinin $(1.0 \mu \mathrm{g} / \mathrm{ml})$, Concanavalin A $(12.5 \mu \mathrm{g} / \mathrm{ml})$, or pokeweek mitogen $(50 \mu \mathrm{g} / \mathrm{ml})$, reconstituted in the above medium. Cultures were incubated at $37^{\circ} \mathrm{C}$ for 72 hours in an atmosphere of $5 \% \mathrm{CO}_{2} /$ air and high humidity, and pulsed with $1.0 \mu \mathrm{Ci}\left({ }^{3} \mathrm{H}\right)$ thymidine 6 hours before harvest. The samples were counted in a Packard Tricarb liquid scintillation spectrophotometer. The results were expressed as stimulation index (SI); in which

$$
\mathrm{SI}=\frac{\text { cells }+ \text { mitogens }(\text { counts } / \mathrm{min})}{\text { cells only }(\text { counts } / \mathrm{min})}
$$

PMNL chemotaxis was performed by the agarose technique. ${ }^{12}$ Torulopsis glabrata activated human serum was used as a source of chemotatic agent. Leucocyte iodination was carried out by the semiautomated method ${ }^{12}$; PMNL bactericidal and fungicidal activity was measured using Staphylococcus aureus $^{12}$ and $T$. glabrata. ${ }^{12}$

Serum level of immunoglobulin classes (IgA, IgG, $\mathrm{IgM})$ and complement components $(\mathrm{C} 3, \mathrm{C} 4)$ were measured by the radial immunodiffusion technique (Behring, West Germany). Total haemolytic complement was measured by determining the amount of complement source needed to produce lysis of $50 \%$ of opsonised sheep red blood cells and expressed as $\mathrm{CN}_{50}$ units $/ \mathrm{ml} .^{15}$

Patients were tested twice, 4 months apart, and results paesented as the mean. During the performance of each test, blood from healthy individuals was included as a control.

\section{Results}

The proportion of 'pitted' red blood cells in 10 normal control patients was less than $1 \%$. None of the 11 children whose spleens had been removed electively had evidence of residual splenic activity on nuclear scan; they had an average of $45 \%$ 'pitted' 
red blood cells (range 33-57\%). Six of the 8 trauma patients had nuclear scan evidence of residual splenic tissue ranging from 2 to $70 \%$ of normal

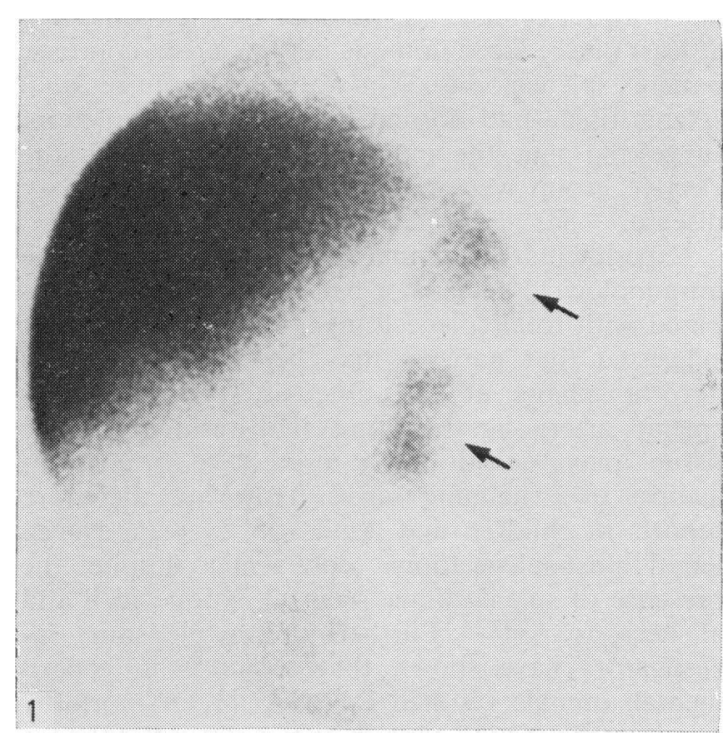

Fig. 1 Left anterior oblique liver/spleen scan of a 21-year-old patient 10 years after splenectomy for traumatic laceration. Residual splenic tissue (arrowed) represents about $20 \%$ normal splenic volume: $15 \%$ of the RBCs were 'pitted'.

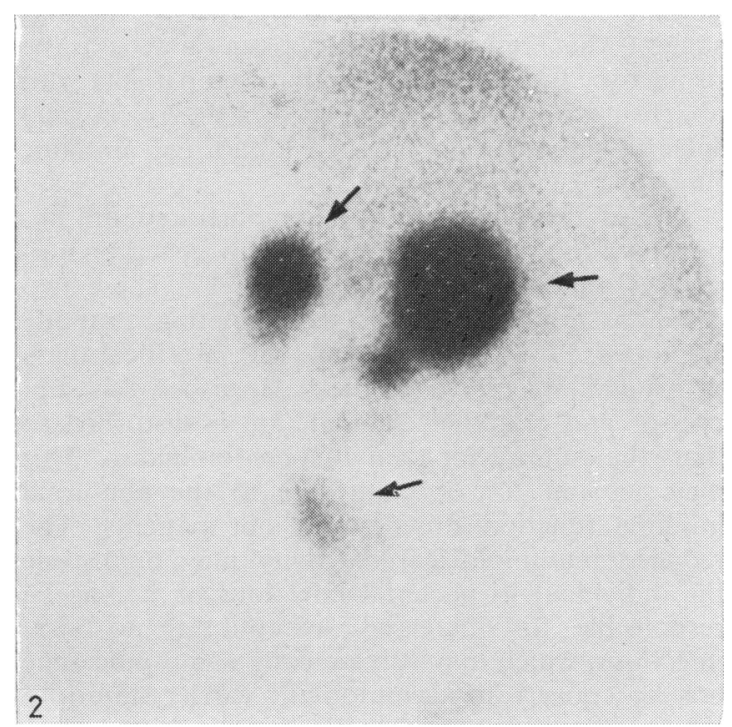

Fig. 2 Left posterior oblique liver/spleen scan of 9-year-old boy 1 year after avulsion, the residual splenic tissue (arrowed) represents about $45 \%$ normal splenic volume: $0.6 \%$ of the RBCs were 'pitted'. splenic volume (Figs 1-2). However, only 4 of the 8 patients showed splenic activity when assessed by percentage of 'pitted' red cells with values of 0.4 , $0 \cdot 6,10$, and $15 \%$. There was no association between the degree of splenosis and the severity of the splenic injury.

Two patients with about 2 and $7 \%$ of normal splenic volume on scan were within the range of the asplenic patients when assessed by percentage of 'pitted' red cells, with values of 54 and $40 \%$ respectively. As the liver/spleen scan was a more sensitive indicator of splenosis the results from this technique were used to determine the relationship between splenosis and immune function.

All patients splenectomised for trauma showed normal levels of T- and B-cells, while 5 of the 11 patients who had undergone elective splenectomy had low T-cell numbers (Fig. 3). The B-cell numbers in the elective splenectomy group were within the normal range.

The patient with approximately $70 \%$ normal splenic volume showed greatly reduced mitogeninduced lymphocyte response to phytohaemagglutinin, pokeweek mitogen, and Concanavalin $A$ with an SI less than 5 , the lower limit of the normal response. In addition, $\operatorname{IgA}(0.53 \mathrm{~g} / \mathrm{l})$ (normal range $=0.8-4.8$ $\mathrm{g} / \mathrm{l})$ and $\operatorname{IgM}(0 \cdot 3 \mathrm{~g} / \mathrm{l}))($ normal range $=0 \cdot 5-2 \cdot 0 \mathrm{~g} / \mathrm{l})$ were below the normal ranges. ${ }^{16}$ This patient developed severe bronchopneumonia 4 years after splenectomy and has also had more frequent minor

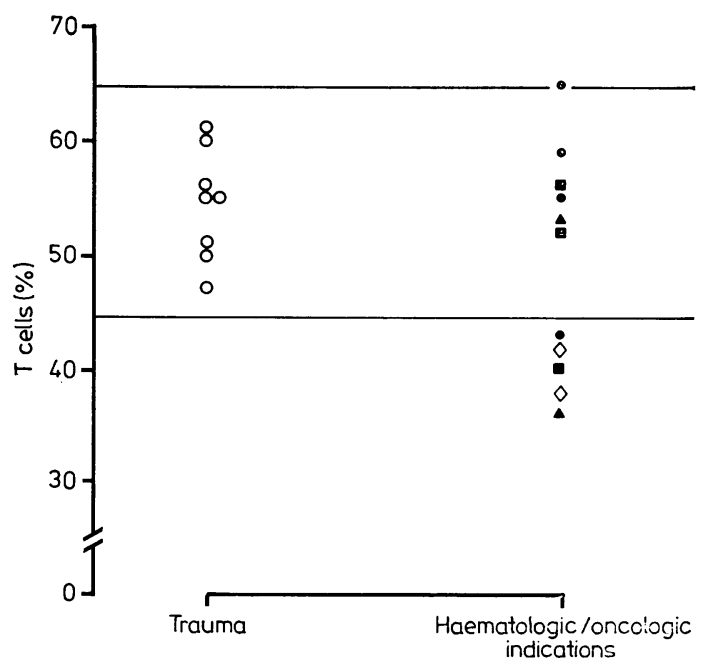

Fig. $3 T$ cell numbers in patients splenectomised for trauma $(O)$ or haematologic/oncologic indications:

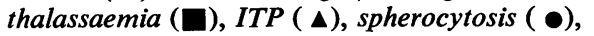
Hodgkin's disease $(\hat{v}) .(-)$ represents normal range. 


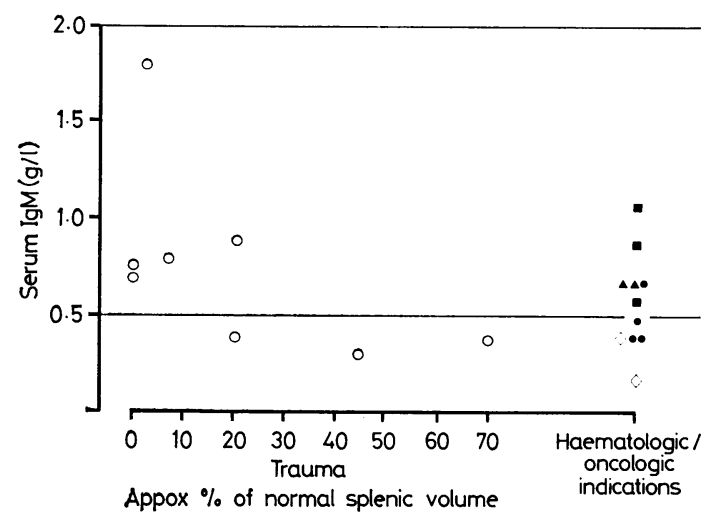

Fig. 4 Serum IgM levels in patients splenectomised for trauma $(\mathrm{O})$ or haematologic/oncologic indications: thalassaemia ( $\mathbf{\square}), \operatorname{ITP}(\Delta)$, spherocytosis $(\bullet)$, Hodgkin's disease $(\hat{v})$. Normal range $(-)$ depicting $2 S D$ concentrations.

upper respiratory tract infections since splenectomy. The other patients in the trauma group demonstrated normal lymphocyte responsiveness to mitogen. Levels of IgM were depressed in all 3 patients who demonstrated the most residual splenic tissue with values of a similar order to the treated Hodgkin's disease patients (Fig. 4). The patient with $45 \%$ normal splenic volume also showed a low level of IgG $(6.6 \mathrm{~g} / \mathrm{l})$; normal range $=7 \cdot 20-19 \cdot 20 \mathrm{~g} / \mathrm{l}$. The remaining patients in both groups did not demonstrate immunoglobulin levels below the normal range.

There was a general depression of lymphocyte responsiveness to mitogen stimulation in patients in the elective group compared with the trauma group. Seven of the 11 patients demonstrated a lymphocyte SI less than 10 in response to at least one of the mitogens and 3 patients ( 2 thalassaemia, 1 spherocytosis) had an SI less than 5, the lower limit of the normal response. Two patients with thalassaemia developed bronchopneumonia, one had 7 episodes of bronchopneumonia in the 4 years after splenectomy, the other also developed $S$. aureus septicaemia 5 years after splenectomy. Both Hodgkin's disease patients developed herpes zoster infections.

Complement levels $\left(\mathrm{C} 3, \mathrm{C} 4\right.$, and $\left.\mathrm{CH}_{50}\right)$ and PMNL functions were normal in both groups of patients.

\section{Discussion}

The increased risk of fulminant and fatal sepsis in splenectomised individuals at all ages, in particular infants and children, has now been established by large population studies. ${ }^{4}$ Individuals who undergo splenectomy for haematologic and oncologic con- ditions have a greatly increased mortality and morbidity owing to sepsis. These clinical observations are substantiated by our finding that $8(73 \%)$ of the 11 children splenectomised for medical reasons and without evidence of residual splenic tissue, had at least one reduced immune parameter. In contrast, patients splenectomised for trauma without residual splenic tissue had normal immunological function, suggesting that the immune abnormalities in the elective group were possibly due to their primary disease.

Experiments on animals have demonstrated the immunological benefits of partial splenectomy compared with total splenectomy. ${ }^{17}$ Such findings have suggested that patients may benefit from splenic autotransplants at the time of splenectomy or splenic artery ligation, and that residual splenic tissue may constitute a protective mechanism against overwhelming sepsis. However, there are reports in children and adults of severe and even lethal post-splenectomy septicaemia despite the presence of splenic tissue seen on isotope scan and at necropsy. ${ }^{4-918}$ Furthermore, while some investigators have shown that splenosis in experimental animals resulted in reduction of mortality rate,$^{19}$ others found that splenosis did not enhance blood stream clearance of pneumococcus, ${ }^{20}$ which is the causative organism in half the cases of overwhelming post-splenectomy infection. ${ }^{4}$ Splenosis is a common occurrence after splenectomy for trauma $^{621}$ and this finding is supported by the present study. Clinical and experimental findings suggest that residual splenic tissue may not be protective. Indeed, in our study three patients with approximately $70 \%, 45 \%$, and $20 \%$ of normal splenic volume demonstrated abnormal immune function.

Mouse experiments have demonstrated an important function of the spleen is to generate suppressor and amplifier lymphocytes. ${ }^{22} 23$ These two functions are important in co-ordinating antibody responses. Possibly, in patients with a critical mass of residual splenic tissue the balance between suppressor and amplifier cell function is disturbed so that the suppressor activity predominates. This may account for or contribute to the abnormal immune function observed in our patients. The reduced immunological reactivity may predispose the individual to bacterial infections or it may be responsible for increased susceptibility to viral infections, which may subsequently compromise the host to convert an asymptomatic carrier state into a fulminant pneumococcaemia. ${ }^{24}$

Autotransplantation of splenic tissue or splenic artery ligation as methods of management of the ruptured spleen have been suggested on the basis of 
animal experiments in order to preserve some of the immunological function of the spleen. The data from our study suggested that information based on these animal studies may not be translated into the human situation.

We thank Mr B S Douglas, Professor G M Maxwell, Professor Y H Thong, Dr K Cheney, Dr J Savage, and Dr I Toogood for help and encouragement and the consultant surgeons of the Adelaide Children's Hospital for access to their patients.

\section{References}

1 Battisto J R, Streilein W, eds. Immuno aspects of the spleen. Amsterdam: Elsevier/North-Holland, 1976.

2 Eichner E R. Splenic function: normal, too much, and too little. Am J Med 1979; 66: 311-20.

3 Spirer Z. The role of the spleen in immunity and infection. Adv Pediatr 1980; 27 : 55-88.

4 Singer D B. Postsplenectomy sepsis. In: Rosenberg H S, Bolande R P, eds. Perspectives in pediatric pathology. Vol. 1. Chicago: Year Book Medical Publishers, 1973: 285-311.

5 Leonard A S, Giebink G S, Baesl T J, Krivit W. The overwhelming postsplenectomy sepsis problem. World $J$ Surg 1980; 4: 423-32.

6 Pearson H A, Johnston D, Smith K A, Touloukian R J. The born again spleen: return of splenic function after splenectomy for trauma. $N$ Engl J Med 1978; 298: 1389-92.

7 Balfanz J R, Nesbit M E, Jr, Jarvis C, Krivit W. Overwhelming sepsis following splenectomy for trauma. J Pediatr 1976; 88: 458-60.

8 Bisno A L, Freeman J C. The syndrome of asplenia, pneumococcal sepsis, and disseminated intravascular coagulation. Ann Intern Med 1970; 72: 389-93.

9 Whitaker A N. Infection and the spleen. Association between hyposplenism, pneumococcal sepsis, and disseminated intravascular coagulation. Med J Aust 1969; i: $1213-9$.

10 Holroyde C P, Oski F A, Gardner F H. The 'pocked' erythrocyte. N Engl J Med 1969; 281 : 516-20.

11 Myers J, Segal R J. Weight of the spleen. I. Range of normal in a nonhospital population. Arch Pathol 1974; 98: 33-8.

12 Ferrante A, Thong Y H. Optimal conditions for simul- taneous purification of mononuclear and polymorphonuclear leucocytes from human blood by the Hypaque-Ficoll method. J Immunol Methods 1980; 36: 109-17.

13 Scheinberg M, Blacklow N R, Goldstein A L, Parrino T A, Rose F B, Cathcart E S. Influenza; response of T-cell lymphopenia to thymosin. N Engl J Med 1976; 294: 1208-11.

14 Alexander E L, Sanders S K. F $\left(\mathrm{ab}^{1}\right)_{2}$ reagents are not required if goat, rather than rabbit, antibodies are used to detect human surface immunoglobulin. J Immunol 1977; 119: 1084-8.

15 Mayer M M. Complement and complement fixation. In: Kabat E A, ed. Experimental immunochemistry, second edition. Springfield, Ill: Thomas, 1971: 133.

16 Beard L J, Thong Y H, Turner T W. The immunological status of children with atopic dermatitis. Acta Paediatr Scand 1981; 70: 551-5.

17 Van Wyck D B, Witte M H, Witte C L, Strunk R C. Humoral immunity in experimental hyposplenism. Surgery 1978; 84: 134-9.

18 Taylor C J. Letter: Recurrent meningitis in a child with combined IgA deficiency and splenic hypoplasia. Arch Dis Child 1981; 56: 486.

19 Likhite V V. Protection against fulminant sepsis in splenectomized mice by implantation of autochthonous splenic tissue. Exp Hematol 1978; 6: 433-9.

20 Schwartz A D, Goldthorn J F, Winkelstein J A, Swift A J. Lack of protective effect of auto-transplanted splenic tissue to pneumococcal challenge. Blood 1978; 51 : 475-8.

21 Spencer G R, Bird C, Prothero D L, Brown T R, Mackenzie F A F, Phillips M J. Spleen scanning with ${ }^{99} \mathrm{Tcm}^{\mathrm{m}}$-labelled red blood cells after splenectomy. $\mathrm{Br} J$ Surg 1981; 68: 412-4.

22 Amsbaugh D F, Prescott B, Baker P J. Effect of splenectomy on the expression of regulatory T-cell activity. $J$ Immunol 1978; 121 : 1483-5.

23 Man-Sun Sy, Miller S D, Kowach H B, Claman H N. A splenic requirement for the generation of suppressor T-cells. J Immunol 1977; 119: 2095-9.

24 Hyslop N E, Jr. Fever and circulatory collapse in an asplenic man. Case records of the Massachusetts General Hospital: No 36, 1975. $N$ Engl J Med 1975: 293: 547-53.

Correspondence to Dr R C Cohen, Department of Surgery, Royal Children's Hospital, Flemington Road, Parkville, Victoria 3052, Australia.

Received 19 January 1982 